\title{
Anomalías anatómicas en anillos de crecimiento anuales de Austrocedrus chilensis (D. Don) Pic.-Serm. et Bizzarri en el norte de su rango de distribución
}

\section{Anatomic anomalies in annual tree-rings of Austrocedrus chilensis (D. Don) Pic.-Serm. et Bizzarri in its northern distribution range}

\author{
Moisés Rojas-Badilla ${ }^{* 1}$, Claudio Álvarez ${ }^{1,2}$, Gonzalo Velásquez-Álvarez ${ }^{1}$, Martín Hadad ${ }^{3}$, Carlos \\ Le Quesne ${ }^{1}$ \& Duncan A. Christie ${ }^{1,2}$
}

'Laboratorio de Dendrocronología y Cambio Global, Instituto de Conservación Biodiversidad y Territorio, Facultad de Ciencias Forestales y Recursos Naturales, Universidad Austral de Chile, Casilla 567, Valdivia, Chile.

${ }^{2}$ Center for Climate and Resilience Research (CR)², Blanco Encalada 2002, $4^{\circ}$ Piso. DGF FCFM. Universidad de Chile, Chile. ${ }^{3}$ Departamento de Dendrocronología e Historia Ambiental, Instituto Argentino de Nivología, Glaciología y Ciencias Ambientales, IANIGLA, C.C. 330,5500 Mendoza, Argentina.

*moisese.rojasbadilla@gmail.com

\begin{abstract}
RESUMEN
Las anomalías anatómicas en los anillos de crecimiento anuales de los árboles han sido poco estudiadas en el sur de Sudamérica, sin embargo son elementos que pueden proporcionar información intra-anual valiosa para el estudio de eventos ambientales. En el presente estudio se aborda la primera caracterización de este tipo de anomalías presentes en anillos de crecimiento en las poblaciones más septentrionales y longevas de Austrocedrus chilensis en Chile central $\left(32^{\circ} \mathrm{S}\right.$ $-35^{\circ} \mathrm{S}$ ). Las tres anomalías descritas fueron: anillos ausentes, bandas intra-anuales y anillos afectados por congelamiento. Los anillos ausentes resultan de la inactividad cambial durante toda una temporada de crecimiento, detectables mediante técnicas de cofechado dendrocronológico. Las bandas intra-anuales se forman a causa de eventos de escases-abundancia de recursos dentro de un mismo periodo de crecimiento y se reconocen por un límite difuso en la madera tardía hacia ambos bordes de la banda. Por último, los anillos por congelamiento son causados por bajas temperaturas y se presentan en el anillo de crecimiento como una línea de células colapsadas. Los porcentajes de presencia de estas anomalías en cada cronología de ancho de anillos relativos a otros estudios indican que $A$. chilensis es propicio para el estudio de anillos ausentes particularmente en su población más septentrional. Las bandas intra-anuales muestran un bajo porcentaje de ocurrencia en la zona de estudio. Finalmente, los anillos por congelamiento se presentan principalmente en edades poco avanzadas de los árboles estudiados en los tres sitios analizados. Estos resultados sugieren realizar posteriores estudios espacio-temporales acerca de la frecuencia de anomalías anatómicas en cronologías de $A$. chilensis a lo largo de su extenso rango de distribución, con el fin de complementar la información ambiental brindada por sus tasas de crecimiento.
\end{abstract}

Palabras clave: anatomía de la madera, anillo ausente, anillo por congelamiento, banda intra-anual, dendrocronología.

\begin{abstract}
Tree-ring anatomical anomalies have received little attention in southern South American trees, however they can contain valuable intra-annual environmental information. This study addressed for the first time the three most frequent treering anomalies recorded in the northern and oldest known Austrocedrus chilensis forest in central Chile (32-35 $\mathrm{S})$. Three anatomic anomalies described were: partially absent rings, intra-annual bands and frost rings. Partially absent rings resulted from cambial inactivity during a complete growing period and require dendrochronological tools to be detected. Intraannual bands are consequence of the abundance-shortage of environmental resources during the growing season and can be detected by examining the undefined late-wood boundaries. Frost rings, are caused by extreme low temperatures and are characterized by collapsed cells in the tree-ring growth. Results indicate that the northern most population exhibited the highest rate of absent rings, while the occurrence of intra-annual bands seems to be rather minor in the study area. Finally, frost rings are registered mainly in the younger trees in all three studied sites. These results suggest the potential for future spatio-temporal studies that examine the frequency of these anatomical anomalies in $A$. chilensis chronologies along its wide geographical distribution. This will complement the current environmental information recorded by its growth rates.
\end{abstract}

KEYwORDs: wood anatomy, absent rings, frost rings, intra-annual bands, dendrochronology. 


\section{INTRODUCCIÓN}

La anatomía vegetal como rama de la botánica se encarga del estudio de la morfología de los tejidos en las plantas y de la formación de anillos anuales en el leño por efecto del crecimiento secundario (Evert 2006). Paralelamente, la dendrocronología es una disciplina científica fuertemente arraigada en la anatomía de la madera y se basa en la comparación de secuencias de anchos de anillos en especies leñosas (Fritts 1976, Schweingruber 1996). Estas secuencias alcanzan una resolución anual para cada período vegetativo, observándose en un corte transversal del leño la estructura de la madera formada generalmente durante las estaciones de primavera y verano. En coníferas, los anillos de crecimiento están formados por células con paredes delgadas y lúmenes amplios al inicio de la temporada de crecimiento representado por una tonalidad de la madera más clara (leño temprano); luego se forman gradualmente paredes celulares más gruesas y lúmenes más estrechos con apariencia más oscura al final de la temporada de crecimiento (leño tardío) (Tortorelli 1956, Roig 1992, Díaz-Vaz 2003). Adicionalmente, existen eventos ambientales que ocurren dentro de la estación de crecimiento y formación de anillos (e.g. sequías, bajas temperaturas, cambios bruscos $\mathrm{y} / \mathrm{o}$ extremos de temperatura y precipitación), que se registran como anomalías que dificultan la correcta identificación de los límites de cada temporada. Las anomalías en los anillos de crecimiento son estructuras anatómicas generadas a partir de un daño celular o formación anormal del tejido xilemático (Schweingruber et al. 1990).

Existen múltiples tipos de anomalías en los anillos de crecimiento anuales (en adelante anillos de crecimiento) de especies leñosas. Entre ellas se pueden mencionar: los anillos parcialmente ausentes (en adelante anillos ausentes), las bandas intra-anuales y los anillos afectados por congelamiento (en adelante anillos por congelamiento). Los anillos ausentes se producen cuando una porción del cambium vascular cesa la producción de tejido durante una temporada completa de crecimiento, como consecuencia de la baja prioridad que el árbol da a la actividad cambial cuando distribuye sus recursos (Schweingruber 1996, Speer 2010). Las bandas intra-anuales son causadas por una variación momentánea de la actividad cambial durante la temporada de crecimiento (Schweingruber 1996), producto de condiciones que limitan severa y momentáneamente el crecimiento, como por ejemplo un período de sequía dentro de la estación de crecimiento, daño mecánico por insectos, viento o fuego, entre otros (Heinrich \& Banks 2006). Finalmente, los anillos por congelamiento están asociados a fenómenos extremos de bajas temperaturas durante el período vegetativo de las plantas leñosas (Kaennel \& Schweingruber 1995).

En el sur de Sudamérica se han desarrollado escasos estudios asociados a la descripción de estructuras anómalas en los anillos de crecimiento, en contraposición a la investigación realizada en el hemisferio norte (St. George et al. 2013, Khishigjargal et al. 2014). Las contribuciones locales se han realizado en la vertiente oriental de los Andes y se centran en la indagación de relaciones entre información climática intra-estacional y la anatomía de los anillos de crecimiento, utilizando especies tales como Nothofagus pumilio (Poepp et Endl.) Krasser, "Lenga" (Masiokas \& Villalba 2004), Araucaria araucana (Molina) K. Koch “Araucaria o Pehuén” (Hadad et al. 2012, Arco et al. 2016) y Austrocedrus chilensis (D. Don) Pic. Serm. et Bizzarri "Ciprés de la cordillera" (Villalba \& Veblen 1996). Por ejemplo, en esta última especie se han encontrado relaciones entre primaveras secas y cálidas seguidas de veranos fríos y húmedos y la aparición de bandas intra-anuales en sus anillos de crecimiento (Villalba \& Veblen 1996).

Austrocedrus chilensis es una conífera dioica endémica del sur de Sudamérica que se distribuye en ambas vertientes de la Cordillera de los Andes entre los 32²9' S y los $43^{\circ} 38^{\prime}$ $\mathrm{S}$, a una altitud entre los 250 y 2200 m s.n.m. (Donoso 1993, Hechenleitner et al. 2005, Roig \& Villalba 2008), pudiendo llegar a alcanzar edades mayores a 1400 años (Le Quesne et al. 2014). En la vertiente occidental de los Andes se ubica su límite de distribución septentrional $\left(32^{\circ} \mathrm{S}\right)$, donde $A$. chilensis crece principalmente en ambientes montañosos $\mathrm{y}$ rocosos, $\mathrm{y}$ en formaciones abiertas con árboles dispersos (Donoso 1982, 2006).

Los anillos de crecimiento de Austrocedrus chilensis son estructuralmente muy bien delimitados, poseen una excelente correlación en el ancho de sus anillos entre árboles de una misma población (cofechado) y son indicadores de la variabilidad ambiental (Schulman 1956). Por estas razones, las cronologías de ancho de anillos de esta especie han sido ampliamente utilizadas en diferentes estudios dendrocronológicos, incluyendo reconstrucciones multicenteniales de precipitaciones (Boninsegna 1988, 2009, Villalba et al. 1998, Le Quesne et al. 2006), de índices de aridez (Christie et al. 2011), de caudales de ríos (Mundo et al. 2012, Muñoz et al. 2016), de historia del fuego (Kitzberger et al. 1997), de eventos tectónicos (Kitzberger et al. 1995) y diversos estudios de dinámica de poblaciones de la especie (Villalba \& Veblen 1997, 1998, Amoroso et al. 2015). Lo anterior ha permitido ampliar considerablemente el conocimiento de la dinámica del clima y la ecología de la especie en Chile central y Patagonia a una escala multicentenial. Debido a la amplia utilidad y excelente calidad que presenta $A$. chilensis como archivo ambiental, y a la escasa información acerca de las anomalías anatómicas presentes en sus anillos de crecimiento, el objetivo de este trabajo es realizar una descripción microscópica y cualitativa de tres tipos de anomalías anatómicas que ocurren en los anillos de crecimiento de esta especie en Chile central. Estas anomalías corresponden a anillos ausentes, bandas intra-anuales y anillos por congelamiento. Además la 
caracterización de este tipo de anomalías contribuiría al conocimiento de la autoecología de $A$. chilensis, y de esta forma aumentar la cantidad de información que se pueda extraer de este valioso archivo ambiental.

\section{MATERIALES Y MÉTODOS}

Área De ESTUdio

Se seleccionaron tres sitios de estudio ubicados en el límite altitudinal de la vegetación arbórea en la zona norte de la distribución de Austrocedrus chilensis entre los $32^{\circ}$ y los $35^{\circ} \mathrm{S}$ (Fig. 1). Los sitios de estudio fueron seleccionados a partir de una extensa red de cronologías de ancho de anillos de la especie desarrollada durante los últimos años a través de su distribución en Chile central (Le Quesne et al. 2014). Dentro de esta red se seleccionaron 3 poblaciones que presentan las siguientes anomalías anatómicas en los anillos de crecimiento, las que se encontrarían ligadas a factores

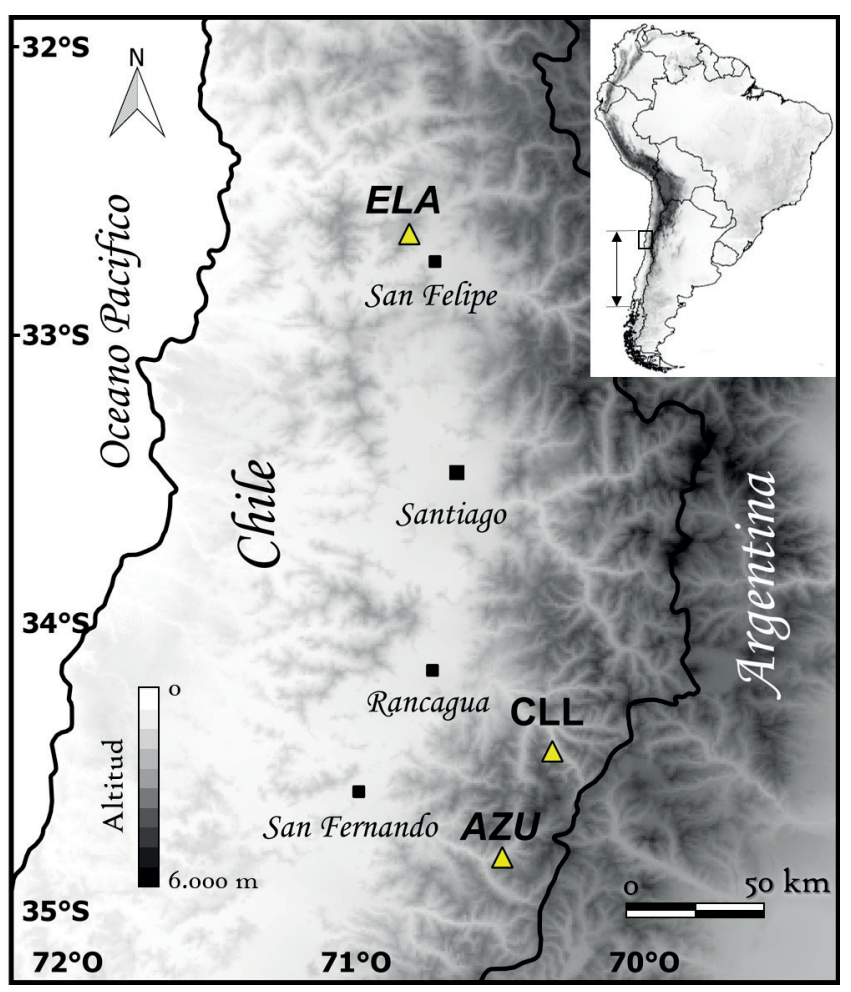

FIgURA 1. Distribución geográfica de Austrocedrus chilensis señalado con flecha negra y localización de los sitios de estudio con triángulos amarillos, de norte a sur: El Asiento (ELA), Cipresillos (CLL) y Río del Azufre (AZU). / Geographic distribution of Austrocedrus chilensis pointed out with black arrow and location of the study sites represented by yellow triangles, from north to south: El Asiento (ELA), Cipresillos (CLL) and Río del Azufre (AZU). ambientales propios de cada sitio: anillos ausentes, bandas intra-anuales, y anillos por congelamiento, las cuales fueron identificados principalmente en los sitios El Asiento (ELA), Cipresillos (CLL) y Río del Azufre (AZU), respectivamente (Fig. 2). Los suelos en el área de estudio corresponden a un sustrato rocoso en pendientes pronunciadas con una escasa retención de agua (Donoso 1993, Donoso et al. 2004). El clima del área de estudio es de tipo Mediterráneo, caracterizado por una sequía estival pronunciada y una concentración $>80 \%$ de la precipitación anual durante los meses de invierno (Miller 1976).

ProcesAmiento DENDROCRONOLÓGICO DE MUESTRAS DE MADERA Y OBTENCIÓN DE FOTOGRAFÍAS

Las muestras de árboles vivos (i.e. testigos de madera) se obtuvieron mediante taladros de incremento que permiten la recolección de testigos de $5 \mathrm{~mm}$ de diámetro, extraídos en un plano transversal y perpendicular al fuste de los árboles. Los testigos se montaron en molduras de madera y se pulieron con lijas de granulometría decreciente (i.e. de la más gruesa a la más fina) según Stokes \& Smiley (1996). Las muestras de madera muerta (i.e. rodelas y cuñas) se extrajeron en su mayoría fragmentadas por la prolongada exposición a los factores ambientales (sol, cambios de temperatura, humedad, viento, etc.), por lo que fueron restauradas en el laboratorio previo al proceso de pulido. Luego, utilizando un microscopio estereoscópico $(<63 \mathrm{x})$ se dataron todas las muestras siguiendo procedimientos dendrocronológicos clásicos (Stokes \& Smiley 1996). Una vez logrado el fechado visual de todas las muestras se midió el ancho de los anillos de crecimiento con una resolución de $0,001 \mathrm{~mm}$ utilizando un carro de medición Velmex. Para comprobar la correcta datación de los anillos de todas las muestras se utilizó el programa COFECHA (Holmes 1983), el cual calcula sucesivas correlaciones móviles entre las mediciones de los anchos de anillos de todas las muestras (series) y determina la posible existencia de errores en el fechado asignándole años calendario a cada anillo de crecimiento. Esto deriva en la construcción de una cronología de anchos de anillos para cada uno de los sitios estudiados también denominada serie maestra.

Para la toma de fotografías se utilizó una cámara Nikon modelo Coolpix p6000 que fue montada en un tercer ocular compatible con el microscopio utilizado para el fechado de las muestras. Todos los segmentos fotografiados tuvieron una correlación mínima de $\mathrm{r}=0,5$, lo que representa un valor confiable para la correcta asignación de años calendario a los anillos de crecimiento con anomalías. Para la composición de fotografías se consideró la disposición de las muestras de izquierda a derecha, en el sentido médula a corteza. 


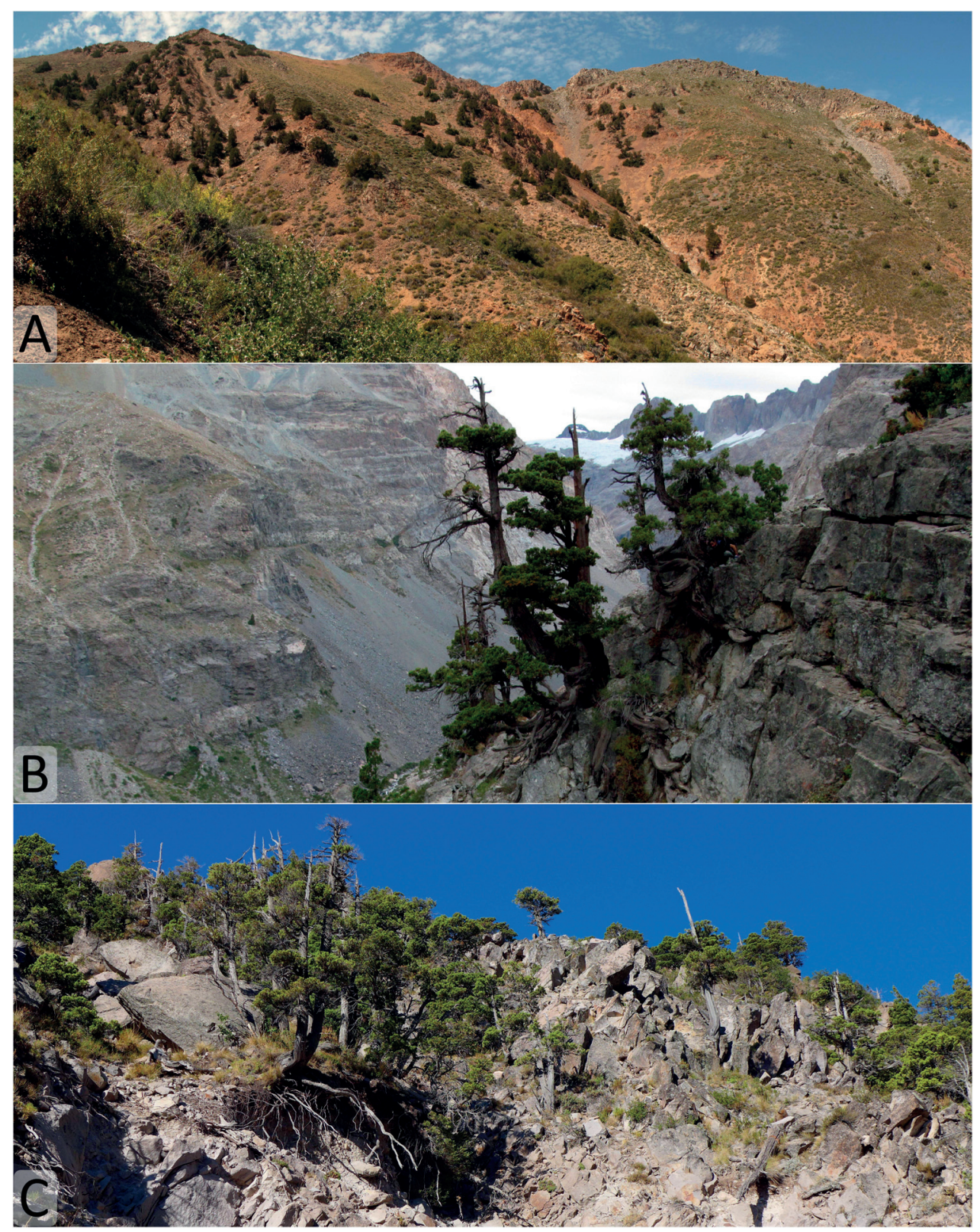

Figura 2. Condiciones de crecimiento en los tres sitios estudiados, donde se observan individuos dispersos de Austrocedrus chilensis creciendo sobre sustrato rocoso. A) El Asiento. B) Cipresillos. C) Río del Azufre. / Growth conditions in the three studied sites, where is shown scattered individuals of Austrocedrus chilensis growing upon rocky cliffs. A) El Asiento. B) Cipresillos. C) Río del Azufre.

\section{RESULTADOS}

Los sitios de El Asiento, Cipresillos y Río del Azufre presentan valores de correlación estadísticamente significativos entre sus series de ancho de anillos, con valores de 0,701, 0,576 y 0,550 respectivamente (Tabla 1). Considerando las muestras de árboles vivos y maderas muertas, el número total de series analizadas para cada sitio fue 123, 118 y 96, respectivamente. De los tres sitios estudiado, la mayor longevidad promedio de las series, el periodo más extenso que cubren las cronologías y el mayor número total de anillos se registró en El Asiento, con un promedio de edad de 449 años, una extensión de la cronología de anillos de crecimiento de 1561 años, y un total de anillos medidos de 55.286 (Tabla 1). 
Tabla 1. Estadísticos descriptivos de las cronologías de los sitios estudiados de Austrocedrus chilensis. *Número de ejemplares vivos de A. chilensis de los que se obtuvo testigos de madera y número total de series medidas. **Número de muestras de madera de árboles muertos y número total de series medidas. ***Intercorrelación entre todas las series medidas por sitio. Es un indicador de la señal común entre los anchos de anillos de los árboles que componen la población. ****Periodo que comprende el registro de anillos en años calendario. / Descriptive statistics of chronologies for the studied sites of Austrocedrus chilensis. ${ }^{*}$ Number of individuals of $A$. chilensis from which wood samples were obtained and total number of series measured. $* *$ Number of wood samples from dead trees and total number of series measured. *** Intercorrelation between all series measured by site. It is an indicator of the common signal between the ring widths of the trees that make up the population. $* * * *$ Period that includes the registration of rings in calendar years.

\begin{tabular}{|c|c|c|c|c|c|c|}
\hline Sitios & $\begin{array}{l}\mathrm{N}^{\circ} \text { ANILlos } \\
\text { TOTALES }\end{array}$ & $\begin{array}{l}\text { No ARBOLES } \\
\text { VIVOS/SERIES* }\end{array}$ & $\begin{array}{c}\mathrm{N}^{\circ} \text { ARBoles MUERTOS/ } \\
\text { SERIES } * *\end{array}$ & EDAD PROMEDIO & CORRELACIÓN $* * *$ & $\begin{array}{c}\text { PERIODO } \\
(\text { AÑOS) } * * * *\end{array}$ \\
\hline El Asiento & 55.286 & $40 / 60$ & $40 / 63$ & $449 \pm 274$ & 0,701 & $451-2011$ \\
\hline Cipresillos & 40.494 & $33 / 51$ & $35 / 67$ & $343 \pm 146$ & 0,576 & $879-2012$ \\
\hline Río del Azufre & 27.181 & $35 / 67$ & $16 / 29$ & $283 \pm 85$ & 0,550 & $1172-2013$ \\
\hline
\end{tabular}

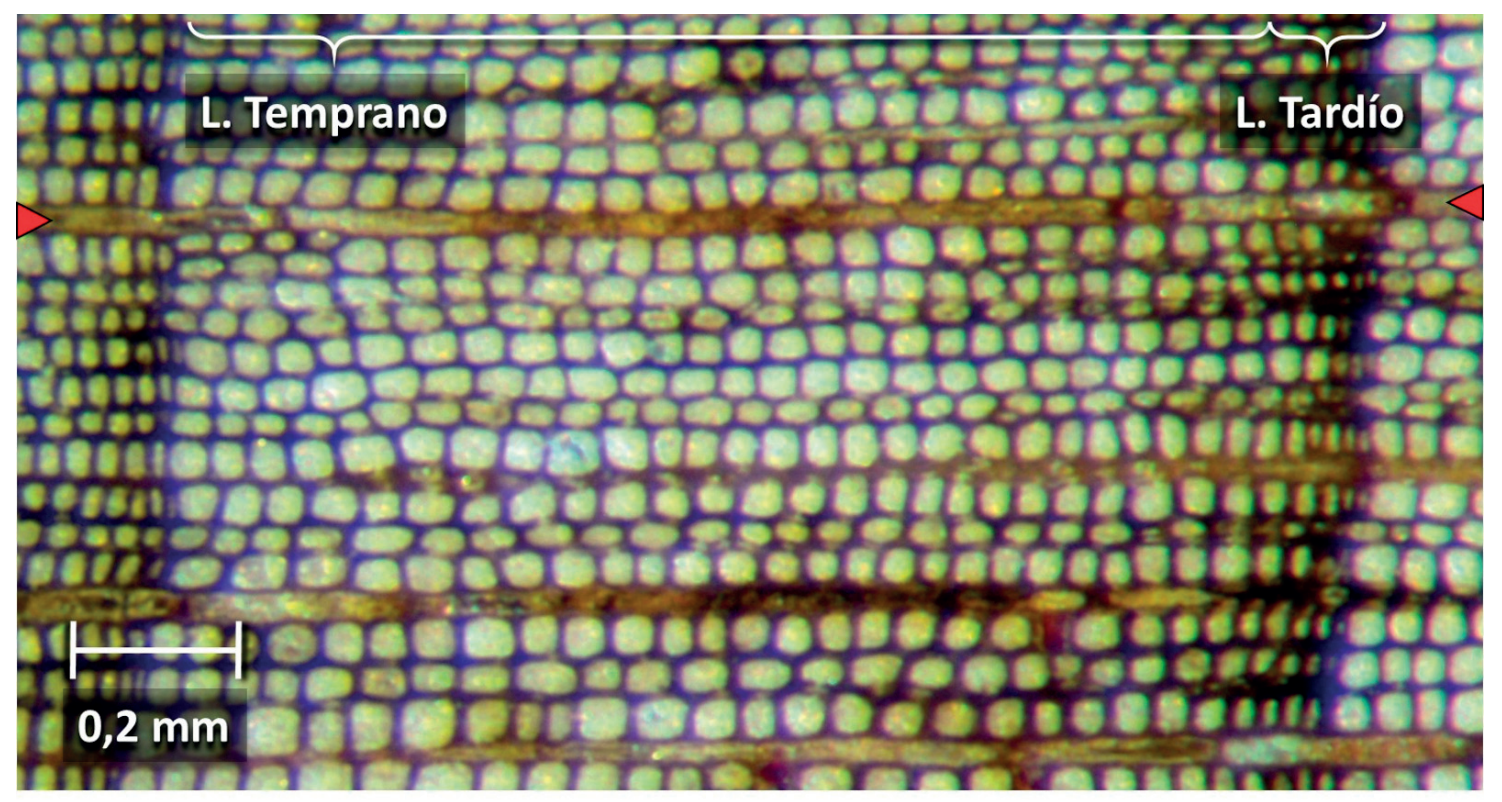

FIgURA 3. Corte transversal de anillo de crecimiento normal de Austrocedrus chilensis, donde se distingue el leño temprano (L. Temprano), el leño tardío (L. Tardío) y los radios medulares perpendiculares al límite de los anillos indicados por los triángulos rojos. / Cross-section of normal tree-ring width of Austrocedrus chilensis, where is distinguished early wood (L. Temprano), late wood (L. Tardío) and wood rays oriented perpendicularly to tree-rings limit indicated by red triangles.

\section{ANILlo TIPO O NORMAL}

En un anillo tipo de Austrocedrus chilensis se distinguen claramente dos zonas: leño temprano y leño tardío (Fig. 3). El leño temprano deriva del reinicio del crecimiento vegetativo a partir de la primavera y resulta del cambio repentino en la tasa de división celular. Se caracteriza por traqueidas grandes de paredes delgadas que a simple vista presentan una tonalidad clara. En contraste, El leño tardío corresponde al crecimiento radial ocurrido hacia el final de la temporada de crecimiento, y exhibe una tonalidad oscura debido al engrosamiento de las paredes celulares y el aplanamiento radial de las células, cuyo límite es marcado por un cambio abrupto en el tamaño y forma de las células entre un año y el siguiente. Como es propio de las Cupressaceae, la proporción de el leño tardío (4-5 células aprox.) es notoriamente menor a el leño temprano (25 células aprox. Fig. 3). También es posible observar los radios leñosos uniseriados con una tonalidad intermedia que cruzan perpendicularmente el leño temprano y el leño tardío. 
CARACTERIZACIÓN DE LAS ANOMALÍAS ANATÓMICAS

Los tres tipos de anomalías descritas están presentes en los tres sitios de estudio con diferentes porcentajes de ocurrencia. La mayor recurrencia de anillos ausentes y el mayor número de series con esta anomalía se registró en El Asiento, seguido de Río del Azufre y Cipresillos (Tabla 2). En cuanto a las bandas intra-anuales, el mayor porcentaje de ocurrencia lo presenta Cipresillos seguido muy por debajo por los otros dos sitios. Por último, los anillos por congelamiento se registran principalmente en las series recolectadas en Río del Azufre, y en menor proporción en los otros dos sitios de estudio.

Además se observó que los anillos ausentes se localizaron generalmente a lo largo de toda la serie temporal independiente de la edad biológica de los individuos. Sin embargo, las bandas intra-anuales y los anillos por congelamiento se presentaron con una mayor regularidad en la porción más joven del leño y son poco frecuentes en edades más avanzadas.
Anillo parcialmente AUSENTE

Los anillos ausentes corresponden a zonas de inactividad del cambium vascular que resulta en la ausencia de crecimiento secundario. En general, los anillos ausentes son detectables de acuerdo al origen de la muestra observada y a sus patrones de crecimiento. Los testigos de madera obtenidos con taladro de incremento abarcan sólo una pequeña porción (radio) del fuste, existiendo una alta probabilidad de interceptar un anillo ausente, detectable sólo en ciertas secciones del tronco. En tal caso, los anillos ausentes sólo se identifican utilizando técnicas de fechado visual (Figs. 4A y 4C) y mediante el programa estadístico COFECHA. Para el caso de rodelas y cuñas (secciones transversales) de árboles muertos fue posible examinar los anillos de crecimiento ausentes a lo largo de toda su extensión, lo cual proporciona una mayor posibilidad de identificar zonas donde el árbol generó tejido celular durante ese periodo vegetativo (Fig. 5).

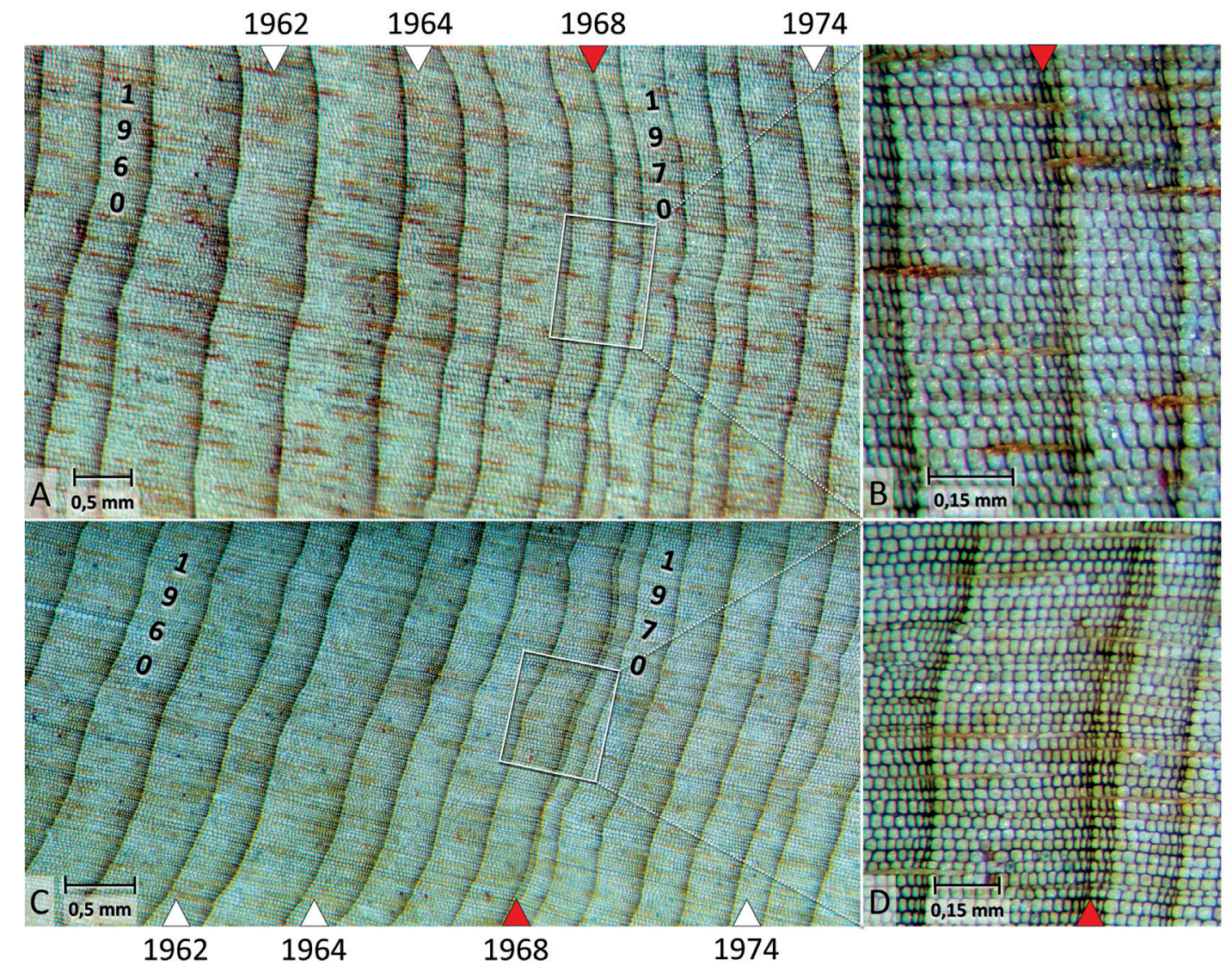

Figura 4. Dos muestras de El Asiento. ELA-162: A) Patrón de crecimiento arbóreo, B) Detalle de anillo ausente. ELA-015, C) Patrón de crecimiento arbóreo, D) Detalle de micro anillo. Secuencia de crecimiento de anchos de anillo que incluye la década de 1960 a 1970. En una de estas secuencias (ELA-162) se puede observar un anillo ausente en el año 1968 (triángulo rojo) parcialmente indistinguible bajo microscopio. El anillo ausente puede ser detectado por la comparación de dos series, utilizando anillos marcadores (triángulos blancos) los cuales definen el patrón de crecimiento de estas dos muestras. / Two samples from El Asiento. ELA-162: A) Tree growth pattern. B) Zoom to absent ring. ELA-015, C) Tree growth pattern. D) Zoom to micro-ring. Tree-ring growth sequence from samples that includes 1960 to 1970 decade. In one of these sequence (ELA-162) is observed an absent ring in the year 1968 (red triangle) partially indistinguishable under microscope. The absent ring can be detected by comparison of two series, using marker rings (white triangle) which defines the tree growth pattern of these two samples. 
TABla 2. Número y porcentaje de series y anillos con anomalías de Austrocedrus chilensis en los sitios estudiados. El porcentaje fue calculado con respecto al número total de anillos para cada sitio (Tabla 1). * Número de anillos y de series representa el valor bruto en donde se detectaron las anomalías respectivamente. ** Los porcentajes son relativos a los anillos y muestras totales de cada cronología. / Number and percentage of series and tree-rings with anomalies of Austrocedrus chilensis in the studied sites. The percentage was calculated with respect to total number of tree-rings for each site. * Number of tree-rings and series represents the raw value where the anomalies were detected respectively. ${ }^{*}$ The percentages are relative to the rings and total samples of each chronology.

\begin{tabular}{|c|c|c|c|c|c|c|}
\hline Sitios & $\begin{array}{l}\text { ANILLOS } \\
\text { AUSENTES } \\
\mathrm{N}^{\circ * / \% * *}\end{array}$ & $\begin{array}{c}\text { SERIES CON } \\
\text { ANILLOS } \\
\text { AUSENTES N }{ }^{\circ * /} \\
\%^{* *}\end{array}$ & $\begin{array}{c}\text { ANILLOS POR } \\
\text { CONGELAMIENTO } \\
\mathrm{N}^{\circ * / \%} \% *\end{array}$ & $\begin{array}{c}\text { SERIES CON } \\
\text { CONGELAMIENTO } \\
\mathrm{N}^{\circ * / \%} \% *\end{array}$ & $\begin{array}{c}\text { ANILLOS CON BANDAS } \\
\text { INTRA-ANUALES } \\
\mathrm{N}^{\circ * / \%} \% *\end{array}$ & $\begin{array}{c}\text { SERIES CON BANDAS } \\
\text { INTRA-ANUALES } \\
\mathrm{N}^{\circ * / \%} \% *\end{array}$ \\
\hline El Asiento & $239 / 0,43$ & $58 / 47,1$ & $84 / 0,15$ & $26 / 21,1$ & $11 / 0,019$ & $7 / 5,69$ \\
\hline Cipresillos & $34 / 0,08$ & $22 / 18,6$ & $144 / 0,35$ & $35 / 29,6$ & $146 / 0,36$ & $47 / 39,80$ \\
\hline Río del Azufre & $37 / 0,13$ & $25 / 26,0$ & $234 / 0,86$ & $56 / 58,3$ & $24 / 0,09$ & $8 / 8,30$ \\
\hline
\end{tabular}

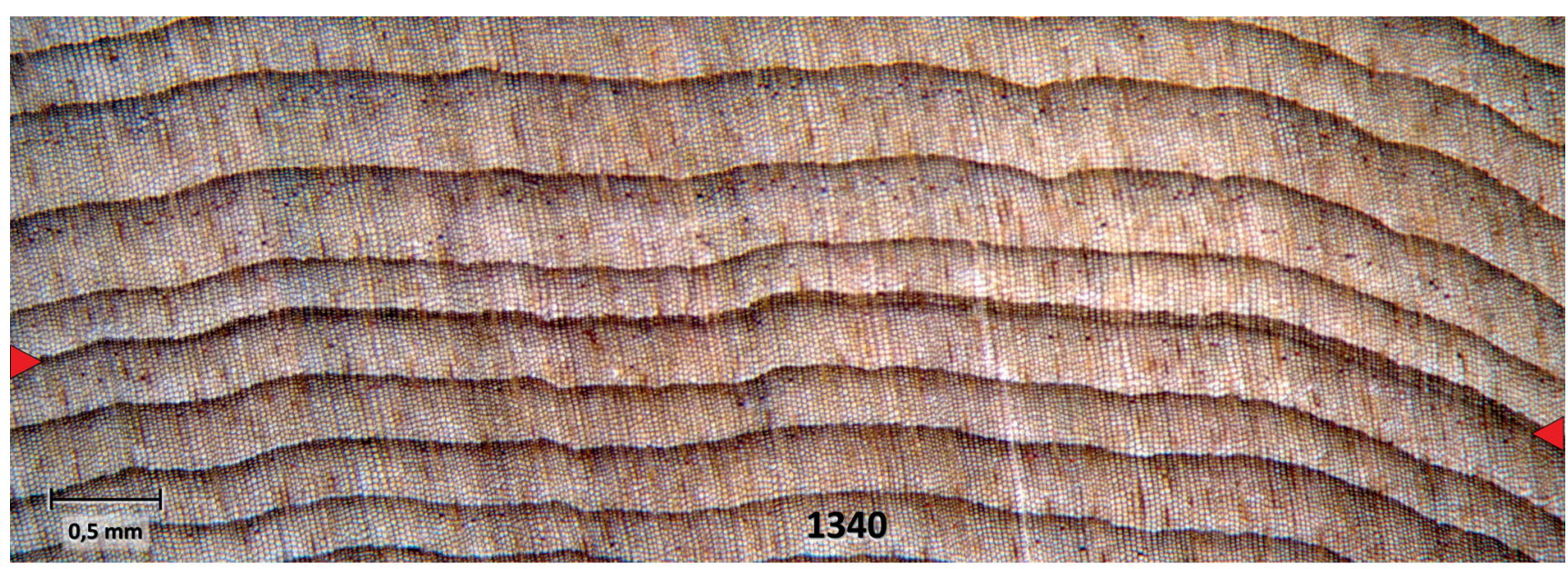

FiguRA 5. Identificación de anillo parcialmente ausente (triángulos rojos) en el año 1344 proveniente de madera relicta recolectada en El Asiento (ELA-055). A la izquierda de la fotografía se observa que el cambium no formó tejido leñoso durante ese año. En contraste, a la derecha se observa una delgada capa de traqueidas. Por ello se refiere a esta anomalía como anillo parcialmente ausente. / Identification of partially absent ring (red triangles) in the year 1344 from relict wood collected in El Asiento (ELA-055). To the left of the photograph shows that the cambium no woody tissue formed during that year. In contrast, to the right shows a thin tracheid layer. Hence that refers to this anomaly as partially absent ring.

\section{BANDA INTRA-ANUAL}

Las bandas intra-anuales se caracterizan por una transición gradual tanto en el tamaño de las células como en el grosor de las paredes celulares en ambos márgenes de la banda (Figs. 6C y D). A primera vista estas bandas parecen ser anillos de crecimiento normales, pero con el fechado visual es posible su detección (Figs. 6A y B). Esta técnica se basa en la comparación entre series de anchos de anillos entre diferentes árboles caracterizadas por un patrón común de años marcadores. Existen testigos de madera de Austrocedrus chilensis en los que estas bandas son relativamente fáciles de detectar, ya sea por la formación anatómica típica de esta anomalía (Fig. 6C) o por la invariabilidad en el ancho de él o los anillos posteriores a la formación de las bandas intra-anuales (Fig. 6B). Por el contrario, cuando se registran anillos ausentes, se produce una disminución en el ancho de los anillos consecutivos a esta anomalía (Masiokas \& Villalba 2004, Le Quesne et al. 2006). También existen situaciones en que resulta compleja la diferenciación de esta anomalía, siendo indispensable usar herramientas digitales como el programa COFECHA.

\section{Anillo AFECTADO POR CONGELAMiENTO}

Esta anomalía se distingue por la presencia de células colapsadas y deformadas dentro de un determinado periodo del desarrollo vegetativo, las que se diferencian del resto del tejido por el gran tamaño de los lúmenes y el delgado grosor de las paredes que presentan las células afectadas, 
produciendo una tonalidad más clara respecto del tejido normal (Fig. 7A). En la Figura 7B se muestran tres anillos por congelamiento en años consecutivos, en todos ellos el tejido afectado se ubica posterior al inicio del periodo de crecimiento, mostrando la variabilidad en la intensidad del daño producido por la helada. También se observa un daño parcial en el año 1317, en donde el tejido contiene aglomeraciones de células colapsadas discontinuas en una matriz de células normales. El anillo siguiente (año 1318) registra células dañadas en casi toda la sección fotografiada, interrumpiéndose en sectores acotados del anillo. En el año 1319 el daño provocado fue continuo observándose a lo largo de todo el anillo, con una delgada separación del tejido leñoso al interior del anillo.
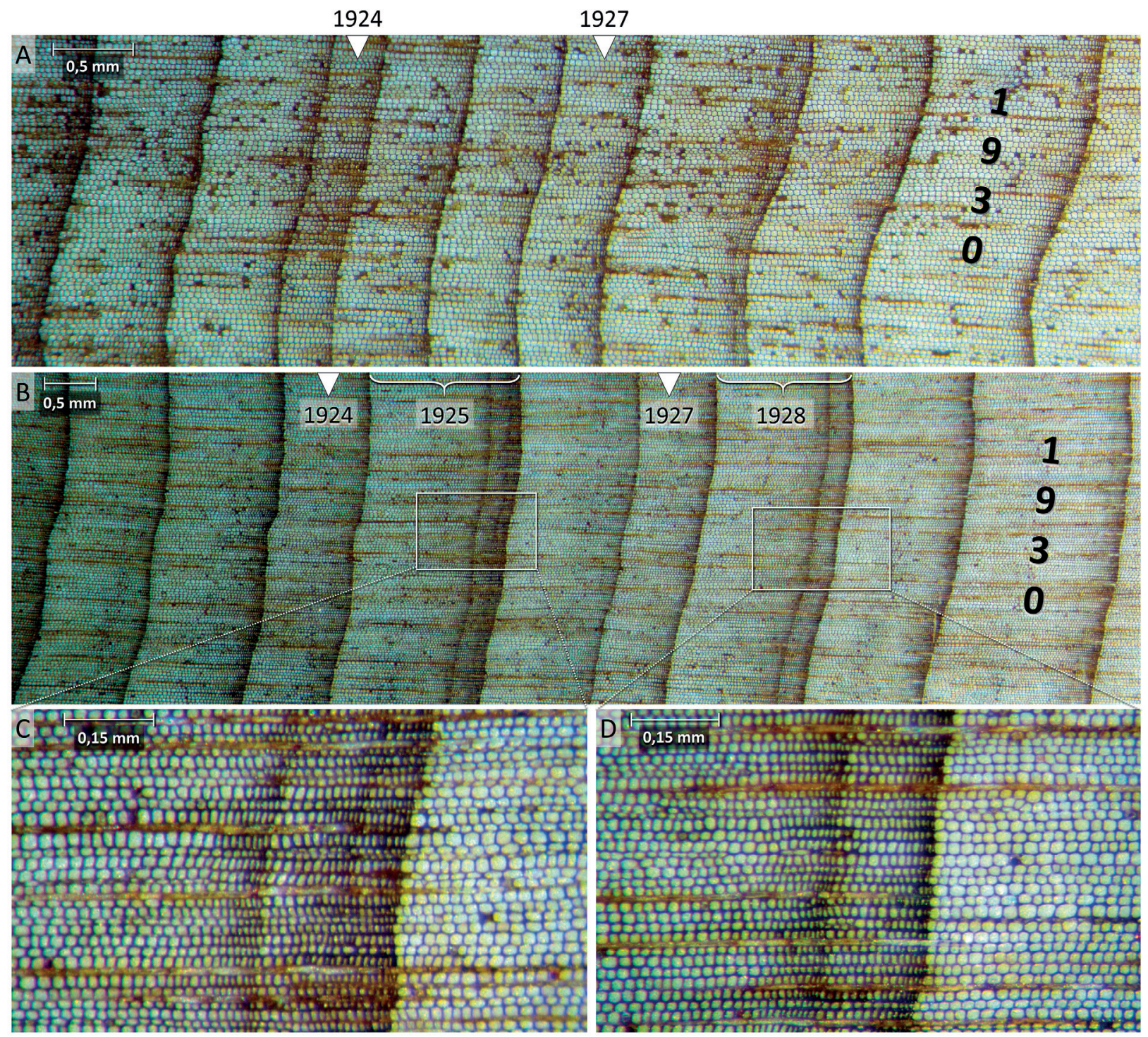

FIGURA 6. Secuencias de anillos de crecimiento con bandas intra-anuales en los años 1925 y 1928 detectadas mediante anillos marcadores (triángulos blancos), en dos muestras de Austrocedrus chilensis recolectadas en Cipresillos. A) Secuencia sin anomalía en el año 1925 con una pequeña porción de leño tardío semejante a una banda intra-anual en el año 1928 (CLL-504A). B) Serie con presencia de dos bandas intra-anuales en los años 1925 y 1928, donde las llaves blancas marcan el ancho real de los anillos (CLL-500A). C) Detalle de banda intraanual presente en el leño tardío del año 1925. D) Detalle de banda intra-anual presente hacia el término del periodo de crecimiento del año 1928. / Tree-ring growth sequences with intra-annual bands in the years 1925 and 1928 detected through marker rings (white triangles), in two samples of Austrocedrus chilensis collected in Cipresillos. A) Sequence without anomalies in the year 1925 with a slight portion of late wood similar to the intra-annual band in the year 1928 (CLL-504A). B) Serie with two intra-annual bands in the year 1925 and 1928 , white keys marks actual tree-ring width (CLL-500A). C) Detail of intra-annual band presents in late wood in the year 1925. D) Detail of intra-annual band presents to the final of growth season in the year 1928. 

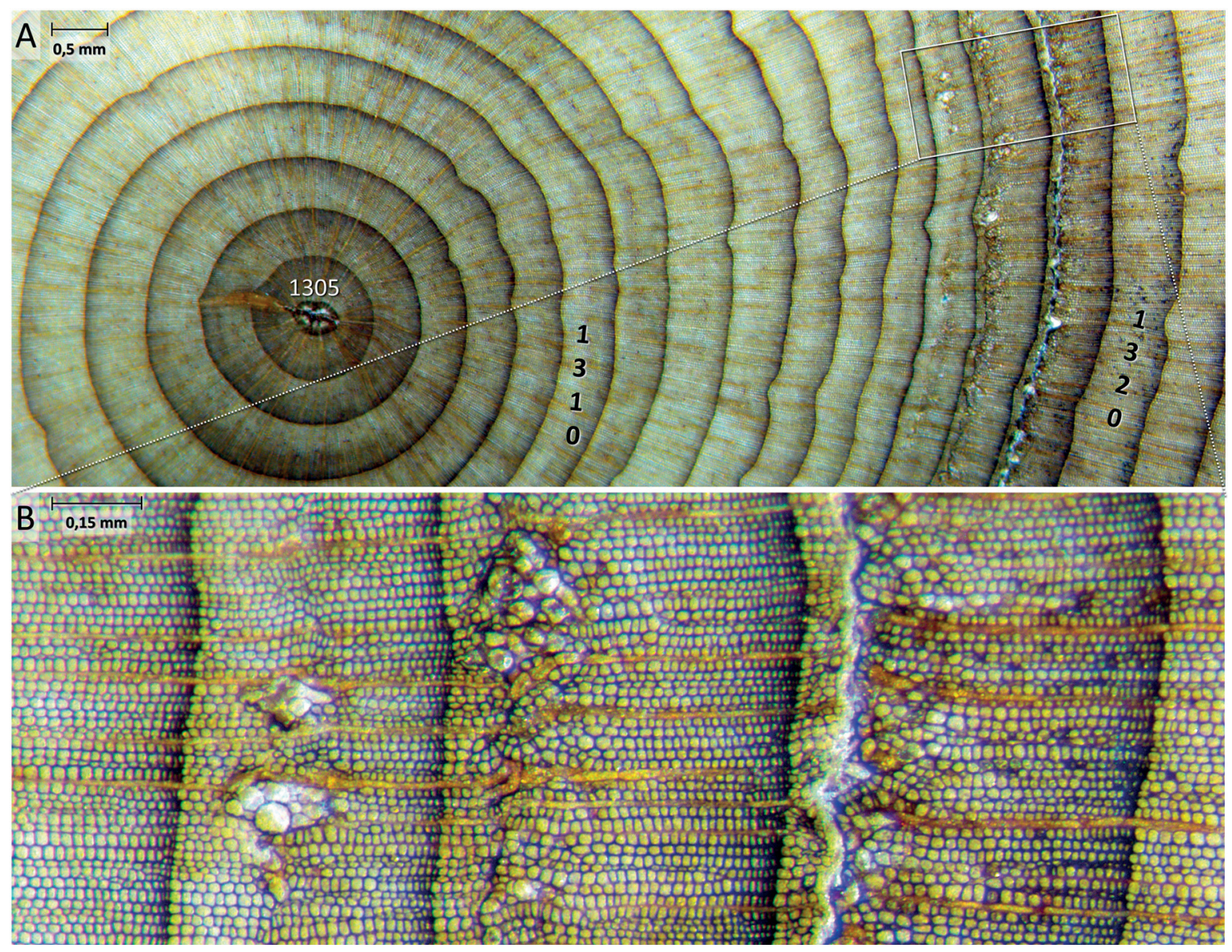

FIgURA 7. Secuencia con tres anillos por congelamiento consecutivos en el año 1317, 1318 y 1319 en una rodela recolectada en Río del Azufre (AZU-113). El primer registro de esta anomalía se presenta a los 13 años, contando desde la médula de la muestra. A) Vista general de las tres primeras marcas de congelamiento. B) Detalle de los tres anillos por congelamiento, con tres diferentes intensidades de daño en el tejido leñoso (xilema). / Tree-ring growth sequence with three consecutive frost rings in the year 1317, 1318 and 1319 in a cross-section from Río del Azufre (AZU-113). The first record of this anomaly is present at 13 years old, counting from the pith sample. A) General view of the three first frost marks. B) Detail of all three frost rings, with three different damage intensity in woody tissue (xylem).

\section{DISCUSIÓN}

Las anomalías pueden dificultar en gran medida la correcta datación de las series de ancho de anillos, asemejándose mucho a límites de anillos "normales" (Figs. 6B y 7A). Sin embargo, cuando se identifican claramente las formaciones anómalas intra-anillo(Figs. 6C, 6D y 7B) es posible utilizarlas como guía para el correcto fechado de las secuencias de anchos de anillos (i.e. como anillos marcadores) (Stahle 1990). Así mismo, es posible detectar qué evento ambiental causó la formación de estas anomalías anatómicas, pudiendo reconstruirlo mediante la comparación entre anomalías, anchos de anillo, reconstrucciones climáticas y registros climáticos instrumentales.
Anillos parcialmente ausentes

La formación de los anillos ausentes ocurre cuando el árbol producto de la escasez de recursos produce fitohormonas de crecimiento insuficientes (principalmente auxina), suprimiendo el inicio del crecimiento, lo que conduce a la inactividad del cambium vascular en una porción del fuste durante toda la temporada de crecimiento (Schweingruber 1996, Savidge 2001, Speer 2010). Las causas de estrés que posiblemente provocan la inhibición del crecimiento pueden ser físicas o bióticas, incluyendo sequías intensas, ataques de insectos, incendios, deslizamientos o avalanchas, y erupciones volcánicas, entre otras. En el caso de los individuos de Austrocedrus chilensis del sitio El Asiento, los anillos parcialmente ausentes están asociados al estrés hídrico (Le Quesne et al. 2006). Cabe destacar que la 
población de A. chilensis de El Asiento es de carácter relictual, formando el límite septentrional y creciendo en la región más árida del rango de distribución de la especie.

El Asiento presenta un porcentaje relativamente alto de anillos ausentes $(0,43 \%)$ (Tabla 2 ) en comparación a cronologías del Hemisferio Norte, donde la mayoría de los valores son $<0,4 \%$ en géneros como Larix $0,2 \%$ y Picea $0,03 \%$ (St. George et al. 2013). Sin embargo, las cronologías del Hemisferio Norte con el mayor porcentaje de esta anomalía (Pinus $0,8 \%$ y Pseudotsuga 0,6\%) (St. George et al. 2013), únicamente son comparables con la población más septentrional de Austrocedrus chilensis que presenta el mayor porcentaje de esta anomalía ya que los valores de los sitios más meridionales de esta especie son mucho más bajos (Cipresillos 0,08\% y Río del Azufre 0,13\%) (Tabla 2). Por tanto, no se podría clasificar a $A$. chilensis como una especie clave para el estudio de anillos ausentes en toda su extensión mediterránea (Chile central), aunque permite analizar las variables relacionadas a esta anomalía en su población más septentrional donde alrededor del $50 \%$ de los individuos presenta algún anillo ausente en sus series de crecimiento.

\section{BANDAS INTRA-ANUALES}

El origen de las bandas intra-anuales se ha relacionado a variaciones climáticas intra-estacionales, como la temprana escasez y posterior aumento de las condiciones favorables para el desarrollo de Austrocedrus chilensis durante su periodo de crecimiento (Villalba \& Veblen 1996, Rozas et al. 2016), al igual que en especies arbóreas del hemisferio norte (Edmonson 2010). Además de las condiciones climáticas que gatillan la presencia de bandas intra-anuales existen diversos factores que interrumpen el crecimiento en otras especies como daños por insectos, enfermedades, viento, fuego (Heinrich \& Banks 2006) y competencia arbórea en bosques densos (Copenheaver et al. 2007).

Austrocedrus chilensis habita en un clima seco que no supera los $500 \mathrm{~mm}$ anuales como lo es el ecotono bosqueestepa patagónica (provincia de Neuquén, Argentina) (Luchini 1981), forma bandas cuando el invierno y principios de primavera son secos y finales de primavera y verano son húmedos (Villalba \& Veblen 1996). Por otra parte, en climas húmedos como el de Valdivia (Chile) en que las precipitaciones generalmente son mayores a 2.400 mm anuales (González-Reyes \& Muñoz 2013), en un suelo con poco drenaje y fuera de su rango de distribución, Rozas et al. (2016) plantean que en una plantación de $A$. chilensis se detectan bandas intra-anuales en coincidencia a un año Niño (seco) previo, seguido de un septiembre lluvioso durante el año de formación del anillo. Esto es debido a un estrés hídrico temprano que luego se revierte con las lluvias de primavera. En ambos casos, esta condición de déficitsuperávit provoca el cese anticipado del crecimiento y la formación de células de leño tardío en el anillo, que luego se reactiva con el periodo húmedo, formando nuevamente células de leño temprano. Asumiendo que esta especie en climas secos y húmedos forma bandas intra-anuales a causa del temprano déficit hídrico durante la estación de crecimiento, se puede inferir que en la zona central de Chile que posee un clima mediterráneo semiárido con sequías y precipitaciones concentradas (Miller 1976), es probable que la misma variación de sequía-humedad sea la causante de esta anomalía.

El sitio de Austrocedrus chilensis que presentó mayor porcentaje de bandas intra-anuales fue Cipresillos con $0,36 \%$ de todos los anillos medidos, ocurriendo en cerca del $40 \%$ de las muestras del sitio. Este valor es menor en comparación a otros estudios del Hemisferio Norte y sur, donde los resultados de Edmonson (2010) muestran un porcentaje de presencia de bandas en cronologías de Juniperus virginiana de 3 y 15\%. En el Arboretum de la Universidad Austral de Chile en Valdivia A. chilensis presenta un 31,8\% de anillos con esta anomalía (Rozas et al. 2016). Por ende el porcentaje de bandas intra-anuales en los sitios analizados en este trabajo es comparativamente bajo. Sin embargo cabe destacar que la población de $A$. chilensis del Arboretum corresponde a una plantación que se ubica fuera del rango de distribución natural de la especie.

Las bandas intra-anuales se pueden utilizar para generar información intra-estacional detallada para los eventos climáticos ya mencionados que influyen en el crecimiento de las especies leñosas. Sin embargo, es esencial contar con una respuesta similar de los individuos a las variaciones ambientales intra-anillo y que esta respuesta coincida con el periodo de registros instrumentales o reconstrucciones climáticas para poder atribuir un origen climático a esta anomalía.

\section{Anillos AFECTAdos POR CONGELAMIENTO}

La ocurrencia de anillos por congelamiento en especies arbóreas ha sido previamente descrita $y$ asociada principalmente a las edades tempranas de los individuos. Estudios en coníferas indican que los individuos jóvenes son los más susceptibles a este tipo de anomalías (Gurskaya \& Shiyatov 2005, Arco et al. 2016). Otro aspecto importante es la ubicación de la marca de congelamiento al interior del anillo (i.e. comienzo, mitad o final del anillo de crecimiento). De lo cual, es posible inferir si los eventos de frío extremo ocurrieron a finales de primavera o principios de verano (Hantemirov et al. 2004). Las reconstrucciones dendrocronológicas de eventos de heladas pueden ser utilizadas para analizar componentes en la variabilidad climática intra-estacional, además de ser empleadas para registrar eventos no climáticos pero que inciden sobre la temperatura como son las erupciones volcánicas (Glerum \& Farral 1966, LaMarche Jr \& Hirschboeck 1984, Hantemirov et al. 2004, Mann et al. 2012). En consecuencia, esta anomalía puede ser utilizada como un indicador complementario de la 
variabilidad climática (Payette et al. 2010).

De los tres sitios de Austrocedrus chilensis incluidos en este estudio, Río del Azufre registró el mayor número de anillos por congelamiento con un total de 234 marcas $(0,86 \%)$ en el $58 \%$ de los individuos (Tabla 2), observándose una baja ocurrencia de esta anomalía en edades biológicas avanzadas de los árboles, coincidiendo con otros resultados en coníferas de Rusia (Gurskaya 2014) y Argentina (Arco et al. 2016). Existen cronologías de anchos de anillos en coníferas del Hemisferio Norte que presentan porcentajes considerables de anillos por congelamiento, por ejemplo Picea mariana (Müll.) Britton, Sterns \& Poggenb. con 28 y 4,3\%, correspondientes al valor más alto y más bajo, respectivamente (Payette et al. 2010). Sin embargo, comparando las cronologías de este estudio con cronologías de la única conífera además de Austrocedrus chilensis que crece en ambientes xéricos en el sur de Sudamérica, Araucaria araucana, los porcentajes de anillos por congelamiento se asemejan. Los sitios de esta especie estudiados por Hadad et al. (2012) se ubican cerca de $350 \mathrm{~km}$ al sur y presentan porcentajes similares de anillos por congelamiento a los que se detectan en A. chilensis creciendo en Río del Azufre. Los porcentajes de anillos con marcas por congelamiento de ambos estudios son, en A. araucana, de 0,81 y $0,18 \%$, correspondiente al sitio más septentrional y meridional, respectivamente, mientras que A. chilensis registró 0,86 y $0,15 \%$ en el sitio más meridional y septentrional respectivamente. Por consiguiente, además de presentar valores cercanos de esta anomalía en ambas especies, los sitios con el mayor porcentaje de anillos por congelamiento son los más cercanos entre sí, el sitio más meridional de $A$. chilensis y el más septentrional de $A$. araucana.

Por lo expuesto anteriormente, es posible considerar a Austrocedrus chilensis como una especie con un buen potencial para el estudio de los anillos de congelamiento en los Andes mediterráneos de Chile a través de la comparación con registros instrumentales y reconstrucciones de temperaturas y precipitaciones. De esta forma se podrían conocer las condiciones climáticas y geográficas que inciden en la ocurrencia de anillos por congelamiento, y desarrollar, en base a este tipo de anomalías, registros de alta resolución temporal de la ocurrencia de eventos de heladas en primavera-verano a escala multicentenial. Además esto permitiría incluir otras especies de coníferas como Araucaria araucana y ampliar la escala espacio-temporal de futuros estudios a objeto de comprender los cambios ambientales ocurridos en el pasado y cómo éstos afectan el crecimiento bajo distintas condiciones para diversas especies del sur de Sudamérica.

ANOMALÍAS ANATÓMICAS COMO INDICADORES AMBIENTALES PARA ESTUdios INTERANUALES EN EL SUR DE SudAmÉRICA

En comparación, el leño tardío de las Cupressaceas del Hemisferio Norte con las sudamericanas es considerablemente más delgada (Roig 1992), lo que limita la realización de cronologías independientes de leño temprano y tardío para inferir por separado las condiciones climáticas pasadas a nivel intra-anual. Sin embargo, los registros de anomalías en coníferas y latifoliadas son indicadores poco estudiados en Sudamérica que permitirían comprender los eventos ambientales a escala intra-anual con el potencial de situarlos espacio-temporalmente. Dichas características posicionan este tipo de estudios como un tópico emergente en la anatomía de la madera y la dendrocronología, lo cual permitiría analizar la variabilidad ambiental pasada con una resolución temporal aún más alta que la actualmente utilizada en las cronologías de ancho de anillos en la región considerada.

\section{AGRADECIMIENTOS}

Agradecemos a CONAF, a la comunidad de El Asiento y a Blanca Tagle por facilitar el acceso a los sitios de estudio; a los arrieros Carlos Farías, Ángel Lara y Kiko Lara, y a Tomás Muñoz, Mariano Masiokas, Mauricio Fuentes, Juan Carlos Aravena, Felipe Benra, Oscar Concha, Daniel Stahle, Jonathan Barichivich, Waldo Iglesias y Maximiliano Le Quesne por su apoyo en terreno. Investigación financiada por el proyecto FONDECYT 1121106.

\section{REFERENCIAS}

Amoroso, M.M., Daniels, L.D., Villalba, R., Cherubini, P. 2015. Does drought incite tree decline and death in Austrocedrus chilensis forests? Journal of Vegetation Science 26: 11711183.

Arco Molina, J.G., Hadad, M.A., Patón, D., Roig, F.A. 2016. Tree age and bark thickness as traits linked to frost ring probability on Araucaria araucana trees in northern Patagonia. Dendrochronologia 37: 116-125.

Boninsegna, J.A. 1988. Santiago de Chile winter rainfall since 1220 as being reconstructed by tree rings. Quaternary of South America and Antarctic Peninsula 6: 67-87.

Boninsegna, J.A., Argollo, J., Aravena, J.C., Barichivich, J., Christie, D., Ferrero, M.E., Lara, A., Le Quesne, C., Luckman, B.H., Masiokas, M., Morales, M., Oliveira, M.J., Roig, F., Srur, A., Villalba, R. 2009. Dendroclimatological reconstructions in South America: A review. Palaeogeography, Palaeoclimatology, Palaeoecology 281: 210-228.

Christie, D.A., Boninsegna, J.A., Cleaveland, M.K., Lara, A., LeQuesne, C., Morales, M.S., Mudelsee, M., Stahle, D.W., Villalba, R. 2011. Aridity changes in the Temperate-Mediterranean transition of the Andes since AD 1346 reconstructed from tree-rings. Climate Dynamics 36: $1505-1521$.

Copenheaver, C.A., Prisley, S.P., Pittman, J.R., Yonce, M.E., Issem, C.M.S., Jensen, K.A. 2007. The geography of grist, flour, and saw mills: Indicators of land use history in 
Virginia. Southeastern Geographer 47: 138-154.

Díaz-VAz, J.E. 2003. Anatomía de maderas. Universidad Austral de Chile, Editorial Marisa Cuneo, Valdivia. 151 pp.

Donoso, C. 1982. Reseña ecológica de los bosques mediterráneos de Chile. Bosque 4: 117-146.

Donoso, C. 1993. Bosques Templados de Chile y Argentina. Variación, estructura y dinámica. Editorial Universitaria. Santiago. 484 pp.

Donoso, C. 2006. Las Especies arbóreas de los Bosques Templados de Chile y Argentina. Autoecología. Marisa Cúneo Ediciones, Valdivia. $678 \mathrm{pp}$.

Donoso, C., Prémoli, A., Gallo, L., Ipinza, R. 2004. Variación intraespecífica en las especies arbóreas de los bosques templados de Chile y Argentina. Editorial Universitaria. Santiago. $420 \mathrm{pp}$.

Edmonson, J.R. 2010. The meteorological significance of false rings in eastern red cedar (Juniperus virginiana L.) from the southern great plain, U.S.A. Tree-Ring Research 66: 19-33.

Evert, R.F. 2006. Esau's Plant Anatomy: Meristems, Cells, and Tissues of the Plant Body: Their Structure, Function, and Development (3rd Edition). John Wiley \& Sons, Inc., New Jersey. $642 \mathrm{pp}$.

FritTs, H.C. 1976. Tree Rings and Climate. Academic Press, New York. 567 pp.

Glerum, C., Farrar, J.L. 1966. Frost ring formation in the stems of some coniferous species. Canadian Journal of Botany 44: 879-886.

González-Reyes, A., Muñoz, A.A. 2013. Cambios en la precipitación de la ciudad de Valdivia (Chile) durante los últimos 150 años. Bosque 4: 191-200.

GurskayA, M.A. 2014. Temperature Conditions of the Formation of Frost Damages in Conifer Trees in the High Latitudes of Western Siberia. Biology Bulletin 41(2): 185-195.

Gurskaya, M.A., Shiyatov, S.G. 2005. Distribution of Frost Injuries in the Wood of Conifers. Russian Journal of Ecology 37(1): 9-15.

Hadad, M.A., Amoroso, M.M., Roig, F. 2012. Frost ring distribution in Araucaria araucana trees from the xeric forests of Patagonia, Argentina. Bosque 33(3): 309-312.

Hantemirov, R.M., Gorlanova, L.A., Shiyatov, S.G. 2004. Extreme temperature events in summer in northwest Siberia since Ad 742 inferred from tree rings. Palaeogeography, Palaeoclimatology, Palaeoecology 209(1-4): 155-164.

Hechenleitner, P., Gardner, M.F., Thomas, P.I., Echeverría, C., Escobar, B., Brownless, P., Martínez, C. 2005. Plantas amenazadas del centro-sur de Chile. Distribución, conservación y propagación. Universidad Austral de Chile y Real Jardín Botánico de Edimburgo, Valdivia. 188 pp.

Heinrich, I., BanKs, J.C.G. 2006. Tree-ring anomalies in Toona ciliata. IAWA Journal 27: 213-231.

HofFer, M., TARDIF, J.C. 2009. False rings in jack pine and black spruce trees from eastern Manitoba as indicators of dry summers. Canadian Journal of Forest Research 39(9): 1722-1736.

Holmes, R.L. 1983. Computer-assisted quality control in tree-ring dating and measurement. Tree-Ring Bulletin 44: 69-78.

Kaennel, M., Schweingruber, F.H. 1995. Multilingual Glossary of Dendrochronology. Haupt Publishers, Berne. 467 pp.

Khishigjargal, M., Dulamsuren, C., Leuschner, H.H., Leuschner, C., Hauck, M. 2014. Climate effects on inter- and intraannual larch stemwood anomalies in the Mongolian forest- steppe. Acta Oecologica 55: 113-121.

Kitzberger, T., Veblen, T.T., Villalba, R. 1995. Tectonic influences on tree growth in northern Patagonia, Argentina. The roles of substrate stability and climatic variation. Canadian Journal of Forest Research 25: 1684-1696.

Kitzberger, T., Veblen, T.T., Villalba, R. 1997. Climatic influences on fire regimes along a rainforest-to-xeric woodland gradient in northern Patagonia, Argentina. Journal of Biogeography 24: 35-47.

LaMarche JR, V.C., Hirschboeck, K.K. 1984. Frost rings in trees as records of major volcanic eruptions. Nature 307: 121126.

Le Quesne, C., Rojas, M., Christie, D. 2014. Anillos de crecimiento de Austrocedrus chilensis: un archivo natural del cambio climático. Revista del Jardín Botánico Chagual 12(1): 3135.

Le Quesne, C., Stahle, D.W., Cleaveland, M.D., Therrell, M., Aravena, J.C., Barichivic, J. 2006. Ancient Austrocedrus tree-ring chronologies used to reconstruct central Chile precipitation variability from AD 1200 to 2000. Journal of Climate 19: 5731-5744.

LuchinI, L. 1981. Estudios ecológicos en la cuenca del río Limay (Argentina). Revista de la Asociación de Ciencias Naturales del Litoral 12: 44-58.

Mann, M.E., Fuentes, J.D., Scott, R. 2012. Underestimation of volcanic cooling in tree-ring based reconstructions of hemispheric temperatures. Nature Geoscience 5: 202-205.

Masiokas, M., Villalba, R. 2004. Climatic significance of intraannual bands in the Wood of Nothofagus pumilio in southern Patagonia. Trees-Structure and Function 18(6): 696-704.

Miller, A. 1976. The climate of Chile. In: Schwerdtfeger, W. (ed.), World survey of climatology, Vol. 12, Climates of Central and South America, pp. 113-145. Ediciones Elsevier, Amsterdam, Netherland.

Mundo, I.A., Masiokas, M.H., Villalba, R., Morales, M.S., Neukom, R., Le Quesne, C., Urrutia, R.B., Lara, A. 2012. Multi-century tree-ring based reconstruction of the Neuquén River streamflow, northern Patagonia, Argentina. Climate of the Past 8: 815-829.

Muñoz, A.A., González-Reyes, A., Lara, A., Sauchyn, D., Christie, D., Puchi, P., Urrutia-Jalabert, R., ToledoGuerrero, I., Aguilera-Betti, I., Mundo, I., Sheppard, P.R., Stahle, D., Villalba, R., Szejner, P., Le Quesne, C., VASTONE, J. 2016. Streamflow variability in the Chilean Temperate-Mediterranean climate transition $\left(35^{\circ} \mathrm{S}-42^{\circ} \mathrm{S}\right)$ during the last 400 years inferred from tree-ring records. Climate Dynamics 47(12): 4051-4066.

Payette, S., Delwaide, A., Simard, M. 2010. Frost-ring chronologies as dendroclimatic proxies of boreal environments. Geophysical Research Letters 37(2): L02711. DOI: 10.1029/2009GL041849

RoIG, F. 1992. Comparative wood anatomy of Southern South American Cupressaceae. IAWA Bulletin 13: 151-162.

Roig, F., Villalba, R. 2008. Understanding climate from Patagonian tree rings. In: Rabassa, J. (ed.), The late Cenozoic of Patagonia and Tierra del Fuego Developments in Quaternary Science, Vol. 11, pp 411-438. Ed. Van Der Meer, Elsevier, Amsterdam, Netherlands.

Rozas, V., Le Quesne, C., Rojas-Badilla, M. 2016. Factores climáticos que controlan el crecimiento radial y la formación de fluctuaciones de densidad en la madera de 
Austrocedrus chilensis en Valdivia, Chile. Bosque 37(3): 461-471

SAVIDGE, R.A. 2001. Intrinsic regulation of cambial growth. Journal of Plant Growth Regulation 20: 52-77.

Schulman, E. 1956. Dendroclimatic Changes in Semiarid America. University of Arizona Press, Tucson. 142 pp.

Schweingruber, F.H. 1996. Tree Rings and environment, Dendroecology. Swiss Federal Institute for Forest, Snow, and Landscape Research, Birmensdorf. 609 pp.

Schweingruber, F.H., Eckstein, D., Serre-Bachet, F., Bräker, O.U. 1990. Identification, presentation and interpretation of event years and pointer years in dendrochronology. Dendrochronologia 8: 9-39.

SpeER, J.H. 2010. Fundamentals of Tree-Ring Research. University of Arizona Press, Tucson. 368 pp.

St. George, S., Ault, T.R., Torbenson, M.C.A. 2013. The rarity of absent growth rings in Northern Hemisphere forests outside the American Southwest. Geophysical Research Letters 40(14): 3727-3731.

Stahle, D.W. 1990. The tree-ring record of false spring in the southcentral USA. Thesis. Doctor of Philosophy. Arizona
State University. Arizona, USA. 272 pp.

Stokes, M.A., Smiley, T.L.1996. An Introduction to Tree-Ring Dating. 2nd ed. University of Arizona Press, Tucson. 73 pp.

Tortorelli, L.A. 1956. Maderas y Bosques Argentinos. Editorial ACME, Buenos Aires. 910 pp.

Villalba, R., Veblen, T.T. 1996. A tree-ring record of dry springset summer events in the forest-steppe ecotone, northern Patagonia, Argentina. In: Dean, J.S., Meko D.M., Swetnam, T.W. (eds.), Tree Rings, Environment, and Humanity, pp. 107-116. University of Arizona, Tucson, USA.

Villalba, R., Veblen, T.T. 1997. Regional patterns of tree population age structures in Northern Patagonia: climatic and disturbance influences. Journal of Ecology 85: 113124.

Villalba R., Veblen, T.T. 1998. Influences of large-scale climatic variability on episodic tree mortality at the forest-steppe ecotone in northern Patagonia. Ecology 79: 2624-2640.

Villalba, R., Cook, E.R., Jacoby, G.C., D’Arrigo, R.D., Veblen, T.T., Jones, P.D. 1998. Tree ring based reconstructions of northern Patagonia precipitation since AD 1600. The Holocene 8(6): 659-674.

Recibido: 24.05.2016

Aceptado: 16.06.2017 